MS34. Growth, structure and

application of multi-component crystals

Chairs: Christopher Frampton, Tomislav Friščić

MS34-01 Form and function of co-crystals: From molecular dating to explosive interactions

Christer B. Aakeröy ${ }^{1}$

1. Department of Chemistry, Kansas State University, Manhattan, KS, 66506, USA

email: aakeroy@ksu.edu

Our ability to imagine and synthesize molecular species has been developed and refined for over a century, and today we are capable of making extraordinary covalent entities that rival some of Nature's best efforts when it comes to structural complexity and chemical reactivity. However, some of the fundamental characteristics of chemistry, communication and change, are primarily initiated and controlled by reversible interactions between molecules, and intermolecular forces provide the conduits through which these interrelated process take place. In this contribution, several strategies for deliberate and directed supramolecular synthesis, based upon systematic structural studies are presented, and some practical applications thereof are also examined.

Keywords: hydrogen bonds, halogen bonds, molecular recognition, energetic materials

\section{MS34-O2 Dynamics of porous metal-organic frameworks}

Len Barbour ${ }^{1}$

1. Department of Chemistry and Polymer Science, University of Stellenbosch, Stellenbosch, South Africa

email: 1jb@sun.ac.za

Metal-organic frameworks are favoured as porous materials owing to their supposed robust structures based on the principle of linking stable secondary-building unit nodes with suitable ligands. However, some frameworks are capable of a surprising level of dynamic behaviour upon activation, heating, guest uptake, and exposure to hydrostatic pressure. This pheomenon has important consequences for the possible utilisation of these materials for guest capture. This presentation will showcase a number of different metal-organic frameworks while discussing their dynamic behaviour in response to external stimuli.

Keywords: MOF, structural dynamics, thermal expansion, gas sorption 